\title{
RETRACTED ARTICLE: Flickr image quality evaluation by deeply fusing heterogeneous visual cues
}

\author{
Yongjun Zheng $^{1} \cdot$ Weiyu $\mathrm{Di}^{1} \cdot$ Shen Jiang $^{2}$
}

Received: 11 October 2017 /Revised: 25 October 2017 / Accepted: 30 October 2017 /

Published online: 23 November 2017

(C) Springer Science+Business Media, LLC, part of Springer Nature 2017

The Editor-in-Chief has retracted this article [1], which was published as part of special issue "Multi-source Weak Data Management using Big Data", because its content has been duplicated without permission from unpublished manuscripts authored by Sandeep Sood and by Pegah Nikbakht, Mojtaba Mahdavi, Shahram Etemadi and Sima Arasteh. The article also shows substantial overlap with a published article [2]. In addition, there is evidence suggesting authorship manipulation and an attempt to subvert the peer review process.

Corresponding author Yongjun Zheng stated on behalf of all co-authors that they agree to this retraction.

\section{References}

1. Zheng, Y., Di, W. \& Jiang, S. Flickr image quality evaluation by deeply fusing heterogeneous visual cues. Multimed Tools Appl (2017). https://doi.org/10.1007/s11042-0175372-8

2. Zhang, L. \& Zimmerman, R. Flickr circles: Mining socially-aware aesthetic tendency. IEEE International Conference on Multimedia and Expo (2015). https://doi.org/10.1109 /ICME.2015.7177384

Electronic supplementary material The online version of this article (https://doi.org/10.1007/s11042-0175372-8) contains supplementary material, which is available to authorized users.

Yongjun Zheng

zhengyongjun1778@126.com

1 College of Metrology and Measurement Engineering, China Jiliang University, Zhejiang, Hangzhou, China

2 School of Journalism \& Communications, Beijing Normal University, Beijing, China 